\title{
A STUDY ON THE QUALITIES THAT AFFECT PREFERENCE OF RURAL RECREATION AREAS
}

\author{
SARI, D. \\ Department of Landscape Architecture, Faculty of Art and Design, Artvin Çoruh University \\ 08000 Arhavi, Artvin, Turkey \\ (e-mail: deryasari@artvin.edu.tr; phone: +90-466-215-1092; fax: +90-466-215-1093)
}

(Received 21 ${ }^{\text {st }}$ Apr 2019; accepted $4^{\text {th }}$ Jul 2019)

\begin{abstract}
Individuals, who struggle due to various stress factors in urban areas, travel to recreation areas to spend their leisure time, to relax, to make contact with nature and to replenish their energy. Rural recreation areas meet these requirements and offer the best natural environment for urban residents. However, the sustainability of recreation areas and recreational activities are important due to the increasing demand and diverse visitor objectives. Thus, sustainable plans for rural recreation areas should include a balance between human preferences and demands and preservation of the nature. The aim of the present study was to determine the qualities that affect the preferences of individuals for rural recreation areas. In this context, the quality preferences of potential visitors in Artvin province (Turkey) were determined based on a survey that investigated 21 qualities in detail. In the study, correlations between participant attributes and preference rates were analyzed with t-test and analysis of variance. The cluster analysis conducted based on the mean preference scores demonstrated that 8 qualities were most effective on preferences. Concurrently, significant differences were determined between occupant attributes and preference scores. The present study was conducted to contribute to future planning strategies for decision makers and further comprehensive studies.
\end{abstract}

Keywords: visitor preferences, rural recreation, survey, quality preferences, nature tourism

\section{Introduction}

Most recreational activities are conducted in natural environments (Kraus, 1971), and today, rural recreation areas are among the spaces where individuals predominantly fulfill their recreational needs. Due to their natural integrity and richness, rural recreation areas offer renewal and leisure opportunities for urban dwellers.

Outdoor recreation refers to the activities that individuals conduct in spaces that allow access to nature or green areas as part of their daily or weekend routines. Nature tourism is a term that includes the activities that individuals conduct to have fun during holidays and focus on nature and generally involve overnight accommodations. This usually means traveling to national parks, forests, lakes, seashores or rural areas and participating in activities that are compatible with the natural qualities of these spaces (Bell et al., 2007).

Outdoor recreation, conducted in natural and semi-natural environments, often referred to as green and blue spaces, plays a crucial role in physical and mental health, as well as wellbeing and social outcomes, and contributes significantly to human health (Hartig et al., 2014; Korpela et al., 2014; Triguero-Mas et al., 2015; Cox et al., 2017; Schirpke et al., 2018). Increasing involvement in outdoor recreation activities can be considered as beneficial for both the individual and the society at large (Eriksson and Nordlund, 2013).

The pressures on individuals to live under negative living conditions in residential areas that are not adequate for their mental and physical requirements, technological advances, transportation facilities and the increase in per capita leisure time improved 
the interest of individuals living in metropolitan cities and high income areas in rural areas (Guo et al., 2010; Sahbaz and Altinay, 2015; Yang, 2017; Schirpke et al., 2018).

Urban parks and green spaces offer physical activity, entertainment, natural experience and social interaction opportunities for urban residents (McCormack et al., 2010; Andkjær and Arvidsen, 2015). For example, ancient trees in these spaces attract visitors and contribute to the urban ecological, aesthetic and functional quality (Jim and Zhang, 2013). However, the activities that individuals could conduct in these spaces, which are part of the urban everyday landscape, are limited. Thus, rural areas play an important role in active outdoor recreation activities for individuals. For example, the main goal in visiting forests is the increasing interest in relaxation, getting away from the daily routine, to exercise and conduct sports activities in the forest (Hansen-Moller and Oustrup, 2004; Andkjær and Arvidsen, 2015).

Nature-based recreation provides visitors the opportunity to meet their recreational needs, while maintaining the diversity and richness of regional natural, cultural and historical resources. Recreational visitors search for natural spaces to meet their recreational needs and often go to remote areas for recreation. This leads to a high demand for natural areas and resources (Shrestha et al., 2007).

As a result of the increase in recreational activities, individuals started to change their recreational space preferences. Thus, the demand for national parks that are rich in natural and cultural values, has increased. Several countries transformed these trends into an advantage, inviting tourists to preservation areas such as national and nature parks (Şahbaz and Altınay, 2015).

The results of surveys conducted in the United States indicated that more than 206 million people aged 15 and over participate in outdoor recreation activities every year, and several outdoor activity participants preferred forests, parks and preservation areas. Visitors visited natural spaces to participate in nature-based recreational activities such as hiking, having fun with family and friends, picnicking and watching natural landscapes, which are among the most popular outdoor activities conducted in the USA (Cordell et al., 2002; Shrestha et al., 2007).

Participation of individuals in recreational activities may vary based on their lifestyles and how they would like to spend their time. The diversity in participation varies based on the country, the social structure, the cultural level and the modern life habits of the population (Tekin et al., 2012). However, several factors such as age, gender, occupation, income level, etc. could be effective on individual recreational preferences. Today, the participation in leisure and recreational activities became a rapid growth industry and the activities offered to masses became a significant market (Tekin et al., 2012).

The extensive literature on outdoor recreation often targeted special natural areas, that include the following elements (De Valck et al., 2017): Forests, mountains, lakes, rivers, coastal areas, protected areas, and national parks. Furthermore, despite the extensive literature on destination attractions (Lee et al., 2010), there is a gap in the literature about studies on the decision-making processes involved in recreational destination preferences for the use of nature (De Valck et al., 2017).

Thus, determination of the factors that affect the demands for nature-human interaction would guide future planners and managers to identify the adequacy, content and adequacy of future recreational activities. The aim of the present study was to determine the qualities that were effective on the selection of rural recreational areas by individuals. 


\section{Materials and methods}

In order to determine the qualities that are effective on rural recreational area preferences, initially, a literature review was conducted. Several studies were found in the literature on nature-based tourism activities. Most accessed research were based on surveys and focused on recreation potential (Plieninger et al., 2013; De Valck et al., 2016) or conducted with proxy-based methods (Paracchini et al., 2014; Grêt-Regamey et al., 2015). Fewer studies emphasized demand based on participant approaches (Beeco et al., 2014; Zoderer et al., 2016) and economic evaluation techniques such as willingness-to-pay (WTP) (Nielsen et al., 2007; Rosenberger et al., 2012) or travel-cost method (Fleming and Cook, 2008; Schirpke et al., 2018). Furthermore, personal interviews with experts and SWOT analysis were also used as an instrument to measure the problems of sustainable tourism in the theoretical framework and to collect qualitative data (e.g. Sanagustin Fons et al., 2011).

The recreational services available in the market were determined based on consumer preferences, that were expressed through demand patterns (Tribe, 2011). Thus, 21 qualities were identified in order to determine the potential visitor expectations from rural recreation areas and the qualities the potential visitors considered more important. The preferences of these qualities were determined with a questionnaire.

The survey was conducted with 448 individuals who were the residents of Artvin province located in northeastern Turkey on the Black Sea coast, Artvin Çoruh University staff and students. In the province of Artvin, there are several rural recreation areas, including 2 National Parks, 3 Nature Preservation Areas, 2 Nature Parks, 2 Natural Monuments, 1 Wildlife Development Area and 1 Biosphere Reserve Area. Furthermore, there are 8 Forest Recreation Areas and 1 Urban Forest (Eminağaoğlu and Beğen, 2015). Due to these characteristics, the survey was conducted in the city of Artvin.

Survey participants were randomly selected and initially, participant demographics such as gender, age, education, profession and income level were determined (Torkildsen, 2005). In the second section of the questionnaire, participants were asked to answer the question "When deciding to visit a rural recreation area, rate your preference for each quality listed below." In the questionnaire, 21 qualities were scored based on a 5-point Likert-type scale. Accordingly, the scoring was as follows: $1=\mathrm{I}$ do not prefer at all, $2=\mathrm{I}$ prefer less, $3=\mathrm{I}$ prefer a little, $4=\mathrm{I}$ prefer a lot, $5=\mathrm{I}$ prefer the most. To improve the comprehensibility of the questions, descriptions (explanations) were provided for Recreation and Rural Recreation Activity terms in the questionnaire form.

Participant demographics and classification were determined with frequency analysis. Mean preference score (MPS) and significance (effectiveness) were determined for each question. For the included qualities, the MPS was classified as follows:

- $1 \leq$ MPS $<2$ : the quality is least effective in rural recreation area preference.

- $2 \leq \mathrm{MPS}<3$ : the quality has moderate effect.

- $3 \leq$ MPS $<4$ : the quality is effective.

- $4 \leq \mathrm{MPS} \leq 5$ : the quality is highly effective.

Cluster analysis was conducted for 21 qualities based on the MPS values. Thus, a dendrogram, where the most preferred qualities were categorized based on the similarities, was created. PAST (Paleontological Statistics) software was used in cluster 
analysis and the dendrogram. The t-test (for gender) and One-Way ANOVA were conducted to determine the significant differences between participant demographics and preferences. The data are presented as a summary table to facilitate the interpretation of the statistical findings. SPSS 19.0 statistics software was used for the analyzes.

\section{Results}

After the survey was conducted, faulty and inconsistent forms were excluded and the answers in the remaining 448 questionnaires were converted into Excel worksheets. Thus, the final 238 participants were male and 210 were female. The distribution and frequencies of the participants based on age (4 categories), education ( 3 categories), occupation (4 categories) and income (6 categories) are presented in Table 1.

Table 1. Survey participant demographics $(n=448)$

\begin{tabular}{c|c|c|c}
\hline \multicolumn{2}{c}{ Participants } & Percentage (\%) & Frequency \\
\hline \multirow{2}{*}{ Gender } & Male & 53.1 & 238 \\
& Female & 46.9 & 210 \\
\hline \multirow{3}{*}{ Age } & $16-25$ & 41.7 & 187 \\
& $26-35$ & 31.7 & 142 \\
& $36-45$ & 19.9 & 89 \\
& $>45$ & 6.7 & 30 \\
\hline \multirow{3}{*}{ Education } & Middle and High school & 14.1 & 63 \\
& University & 63.8 & 286 \\
& Postgraduate & 22.1 & 99 \\
\hline \multirow{3}{*}{ Occupation (Job) } & Student & 33.9 & 152 \\
& Civil servant & 29.5 & 76 \\
& Self-employment/Private sector employee & 17.0 & 88 \\
\hline \multirow{3}{*}{ Income* (TL) } & Other (unemployed, worker, retired, etc.) & 19.6 & 150 \\
& No income & 33.5 & 52 \\
& 500-1500 & 11.6 & 51 \\
& $1600-2500$ & 11.4 & 53 \\
& $2600-3500$ & 11.8 & 74 \\
& $3600-4500$ & 16.5 & 68 \\
\hline
\end{tabular}

*1 Turkish lira $(\mathrm{TL})=0.16 \mathrm{EUR}=0.18 \mathrm{USD}$, The cross exchange rate is based on the date 18.03. 2019

Based on the survey data, where 21 effective qualities on rural recreational area preferences of individuals were questioned, arithmetic mean of preference scores was determined for each question (Table 2). The general study findings demonstrated that the four most preferred qualities were "being in contact with the nature, facilities to rest, relaxation, and to have a peace of mind" $(\mathrm{Q} 3$, MPS $=4.68)$, "Recreation area has beautiful landscapes" $(\mathrm{Q} 4, \mathrm{MPS}=4.58)$, "The recreational area is clean and natural" $(\mathrm{Q} 11, \mathrm{MPS}=4.58)$, and "The recreation area is safe" (Q20, MPS = 4.49). The three least preferred qualities were "availability of activities such as festivals, concerts etc. in 
the recreation area" $(\mathrm{Q} 18, \mathrm{MPS}=3.12)$, "Presence of various geological formations in the recreation area" $(\mathrm{Q} 15, \mathrm{MPS}=3.60)$ and "a space I have experienced and enjoyed before" (Q21, MPS = 3.78).

Table 2. The qualities that were effective on rural recreational area preferences and mean preference scores (MPS)

\begin{tabular}{|c|c|c|}
\hline Questions/qualities & Qualities that affect rural recreational area preferences & MPS \\
\hline Q1 & Easy access to the recreation area & 3.98 \\
\hline Q2 & Inexpensive recreational area & 3.94 \\
\hline Q3 & $\begin{array}{l}\text { Being in contact with the nature, facilities to rest, relaxation, and to have a peace of } \\
\text { mind }\end{array}$ & 4.68 \\
\hline Q4 & Recreation area has beautiful landscapes & 4.58 \\
\hline Q5 & The area allows the visitors to leave with good memories & 4.40 \\
\hline Q6 & The area provides entertainment facilities & 3.96 \\
\hline Q7 & The area makes the visitors feel renewed physically and psychologically & 4.43 \\
\hline Q8 & $\begin{array}{c}\text { Recreation area facilities that allow the visitors to spend time with family and } \\
\text { friends }\end{array}$ & 4.42 \\
\hline Q9 & Availability of facilities that allow to spend alone time in the recreational area & 3.83 \\
\hline Q10 & The area provides different landscapes in summer, winter and fall & 4.29 \\
\hline Q11 & The recreational area is clean and natural & 4.58 \\
\hline Q12 & The area provides food-beverage and picnic facilities & 4.08 \\
\hline Q13 & $\begin{array}{l}\text { The area provides facilities for sports activities (such as skiing, swimming, trekking, } \\
\text { mountain biking, etc.) }\end{array}$ & 3.90 \\
\hline Q14 & $\begin{array}{c}\text { Availability of moving or still water in the recreation area (such as lake, sea or } \\
\text { stream) }\end{array}$ & 4.03 \\
\hline Q15 & $\begin{array}{l}\text { Presence of various geological formations in the recreation area (such as valleys, } \\
\text { canyons, and cliffs) }\end{array}$ & 3.60 \\
\hline Q16 & Presence of a forest in the recreation area & 4.10 \\
\hline Q17 & Calm and non-crowded recreation area & 4.05 \\
\hline Q18 & $\begin{array}{l}\text { Organization of activities such as festivals, festivities, concerts, etc. in the } \\
\text { recreational area }\end{array}$ & 3.12 \\
\hline Q19 & Facilities to experience adventures and discoveries & 3.90 \\
\hline Q20 & Safety in the recreation area & 4.49 \\
\hline Q21 & Previously experienced and satisfactory recreational area & 3.78 \\
\hline
\end{tabular}

The groups formed as a result of the cluster analysis, where the preferred qualities were classified based on similarity, are presented in Figure 1.

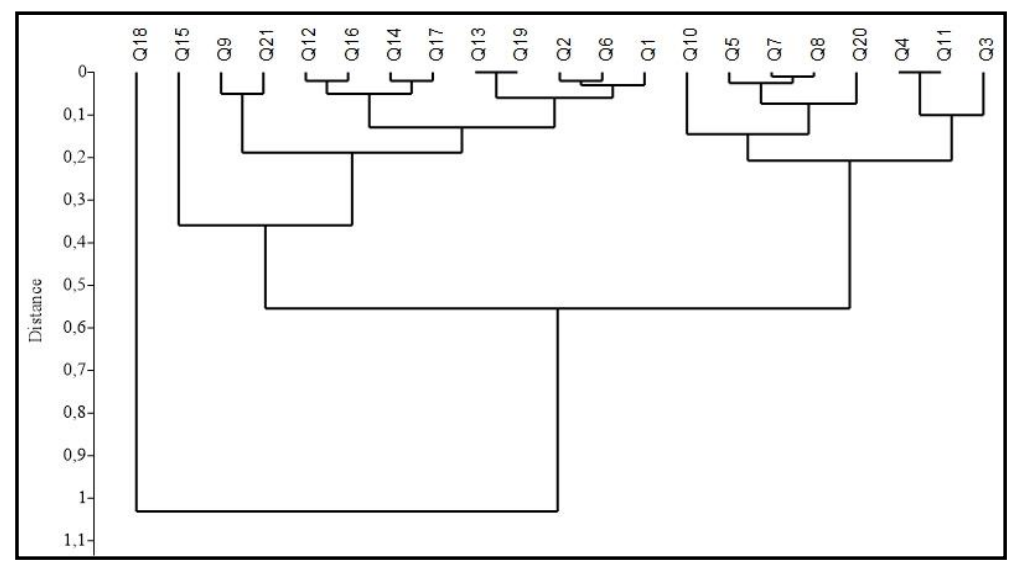

Figure 1. Cluster analys dendrogram for qualities (Similarity measure: Euclidean) 
Thus, the most effective qualities about rural recreation area preferences were grouped as follows:

- Q3: "Being in contact with the nature, facilities to rest, relaxation, and to have a peace of mind".

- Q11: "The recreational area is clean and natural".

- Q4: "Recreation area has beautiful landscapes".

- Q20: "The recreation area is safe".

- Q8: "Recreation area facilities that allow the visitors to spend time with family and friends".

- Q7: "The area makes the visitors feel renewed physically and psychologically".

- Q5: "The area allows the visitors to leave with good memories".

- Q10: "The area provides different landscapes in summer, winter and fall".

T-test and analysis of variance were used to determine whether there were significant differences based on gender, age, education, occupation and income. It was determined that there were significant differences between preference scores of Q1, Q3, Q4, Q5, Q6, Q7, Q8, Q9, Q11, Q20 based on gender (Table 3), Q2, Q3, Q6, Q9, Q16, Q18, Q19 based on age (Table 4), Q2, Q3, Q4, Q6, Q7, Q8, Q9, Q12, Q16, Q18 based on education level (Table 5), Q2, Q5, Q9, Q13 based on occupation (Table 6), and Q2, Q5, Q6, Q7, Q9, Q12, Q16, Q18, Q21 based on income level (Table 7).

Table 3. Mean preference and significance levels of questioned qualities based on participant gender

\begin{tabular}{c|c|c|c|c}
\hline \multirow{2}{*}{ Qualities } & \multicolumn{3}{|c}{ Gender } \\
\cline { 2 - 5 } Q1 & Male & Female & F & Sig. \\
Q2 & $3.88 \mathrm{a}$ & $4.10 \mathrm{~b}$ & 0.923 & 0.035 \\
Q3 & $3.86 \mathrm{a}$ & $4.04 \mathrm{a}$ & 4.515 & 0.055 \\
Q4 & $4.61 \mathrm{a}$ & $4.76 \mathrm{~b}$ & 16.756 & 0.015 \\
Q5 & $4.47 \mathrm{a}$ & $4.70 \mathrm{~b}$ & 24.832 & 0.001 \\
Q6 & $4.27 \mathrm{a}$ & $4.54 \mathrm{~b}$ & 8.86 & 0 \\
Q7 & $3.82 \mathrm{a}$ & $4.11 \mathrm{~b}$ & 0.461 & 0.003 \\
Q8 & $4.29 \mathrm{a}$ & $4.58 \mathrm{~b}$ & 15.819 & 0 \\
Q9 & $4.32 \mathrm{a}$ & $4.52 \mathrm{~b}$ & 3.793 & 0.011 \\
Q10 & $3.72 \mathrm{a}$ & $3.95 \mathrm{~b}$ & 1.939 & 0.028 \\
Q11 & $4.22 \mathrm{a}$ & $4.36 \mathrm{a}$ & 1.431 & 0.095 \\
Q12 & $4.52 \mathrm{a}$ & $4.65 \mathrm{~b}$ & 11.27 & 0.048 \\
Q13 & $4.04 \mathrm{a}$ & $4.13 \mathrm{a}$ & 0.007 & 0.318 \\
Q14 & $3.86 \mathrm{a}$ & $3.94 \mathrm{a}$ & 0.429 & 0.423 \\
Q15 & $3.99 \mathrm{a}$ & $4.07 \mathrm{a}$ & 0.503 & 0.416 \\
Q16 & $3.66 \mathrm{a}$ & $3.54 \mathrm{a}$ & 0.386 & 0.255 \\
Q17 & $4.11 \mathrm{a}$ & $4.09 \mathrm{a}$ & 0.002 & 0.753 \\
Q18 & $4.02 \mathrm{a}$ & $4.08 \mathrm{a}$ & 1.342 & 0.57 \\
Q19 & $3.03 \mathrm{a}$ & $3.23 \mathrm{a}$ & 0.032 & 0.105 \\
Q20 & $3.85 \mathrm{a}$ & $3.95 \mathrm{a}$ & 0.019 & 0.367 \\
Q21 & $4.34 \mathrm{a}$ & $4.66 \mathrm{~b}$ & 26.217 & 0.162 \\
\hline
\end{tabular}

Letters $a, b$ denote similarities and differences based on t-test. Same letter denotes similarity, different letters denote difference 
Table 4. Mean preference and significance levels of questioned qualities based on participant age

\begin{tabular}{|c|c|c|c|c|c|c|}
\hline \multirow{2}{*}{ Qualities } & \multicolumn{6}{|c|}{ Age } \\
\hline & $16-25$ & $26-35$ & $36-45$ & $>45$ & $\mathbf{F}$ & Sig. \\
\hline Q1 & $4.01 \mathrm{a}$ & $4.06 \mathrm{a}$ & $3.80 \mathrm{a}$ & $3.97 \mathrm{a}$ & 1.211 & 0.305 \\
\hline Q2 & $4.22 \mathrm{c}$ & $3.87 \mathrm{~b}$ & $3.48 \mathrm{a}$ & $3.93 \mathrm{bc}$ & 11.677 & 0 \\
\hline Q3 & $4.71 \mathrm{ab}$ & $4.77 \mathrm{~b}$ & $4.49 \mathrm{a}$ & $4.67 \mathrm{ab}$ & 3.543 & 0.015 \\
\hline Q4 & $4.55 \mathrm{a}$ & $4.62 \mathrm{a}$ & $4.58 \mathrm{a}$ & $4.53 \mathrm{a}$ & 0.314 & 0.815 \\
\hline Q5 & $4.48 \mathrm{a}$ & $4.35 \mathrm{a}$ & $4.35 \mathrm{a}$ & $4.27 \mathrm{a}$ & 1.195 & 0.311 \\
\hline Q6 & $4.15 \mathrm{~b}$ & $3.89 \mathrm{ab}$ & $3.69 \mathrm{a}$ & $3.90 \mathrm{ab}$ & 4.582 & 0.004 \\
\hline Q7 & $4.42 \mathrm{a}$ & $4.51 \mathrm{a}$ & $4.35 \mathrm{a}$ & $4.27 \mathrm{a}$ & 1.238 & 0.295 \\
\hline Q8 & $4.41 \mathrm{a}$ & $4.46 \mathrm{a}$ & $4.33 \mathrm{a}$ & $4.50 \mathrm{a}$ & 0.59 & 0.622 \\
\hline Q9 & $3.89 \mathrm{ab}$ & $3.66 \mathrm{a}$ & $3.85 \mathrm{ab}$ & $4.17 \mathrm{~b}$ & 2.137 & 0.095 \\
\hline Q10 & $4.32 \mathrm{a}$ & $4.23 \mathrm{a}$ & $4.18 \mathrm{a}$ & $4.70 \mathrm{~b}$ & 3.01 & 0.03 \\
\hline Q11 & $4.56 \mathrm{a}$ & $4.59 \mathrm{a}$ & $4.56 \mathrm{a}$ & $4.67 \mathrm{a}$ & 0.221 & 0.882 \\
\hline Q12 & $4.14 \mathrm{a}$ & $4.03 \mathrm{a}$ & $4.01 \mathrm{a}$ & $4.13 \mathrm{a}$ & 0.601 & 0.615 \\
\hline Q13 & $4.03 \mathrm{a}$ & $3.77 \mathrm{a}$ & $3.75 \mathrm{a}$ & $4.03 \mathrm{a}$ & 2.351 & 0.072 \\
\hline Q14 & $4.07 \mathrm{a}$ & $3.97 \mathrm{a}$ & $3.97 \mathrm{a}$ & $4.17 \mathrm{a}$ & 0.622 & 0.601 \\
\hline Q15 & $3.56 \mathrm{a}$ & $3.58 \mathrm{a}$ & $3.63 \mathrm{a}$ & $3.90 \mathrm{a}$ & 0.872 & 0.455 \\
\hline Q16 & $4.03 \mathrm{a}$ & $4.12 \mathrm{ab}$ & $4.12 \mathrm{ab}$ & $4.40 \mathrm{~b}$ & 1.473 & 0.221 \\
\hline Q17 & $4.00 \mathrm{a}$ & $4.04 \mathrm{a}$ & $4.11 \mathrm{a}$ & $4.17 \mathrm{a}$ & 0.387 & 0.762 \\
\hline Q18 & $3.40 \mathrm{c}$ & $3.12 \mathrm{bc}$ & $2.67 \mathrm{a}$ & $2.77 \mathrm{ab}$ & 7.383 & 0 \\
\hline Q19 & $4.06 \mathrm{~b}$ & $3.87 \mathrm{ab}$ & $3.61 \mathrm{a}$ & $3.83 \mathrm{ab}$ & 3.58 & 0.014 \\
\hline Q20 & $4.48 \mathrm{a}$ & $4.46 \mathrm{a}$ & $4.55 \mathrm{a}$ & $4.57 \mathrm{a}$ & 0.318 & 0.812 \\
\hline Q21 & $3.81 \mathrm{a}$ & $3.67 \mathrm{a}$ & $3.90 \mathrm{a}$ & $3.70 \mathrm{a}$ & 0.981 & 0.401 \\
\hline
\end{tabular}

Letters $a, b, c$ denote similarities and differences based on variance analysis. Same letter denotes similarity, different letters denote difference (Post Hoc Test, Duncan, alpha 0.05)

Table 5. Mean preference and significance levels of questioned qualities based on participant education

\begin{tabular}{c|c|c|c|c|c}
\hline \multirow{2}{*}{ Qualities } & \multicolumn{5}{|c}{ Education } \\
\cline { 2 - 6 } & Middle and High school & University & Postgraduate & F & Sig. \\
\hline Q1 & $4.05 \mathrm{a}$ & $3.97 \mathrm{a}$ & $3.97 \mathrm{a}$ & 0.138 & 0.871 \\
Q2 & $4.19 \mathrm{~b}$ & $4.01 \mathrm{~b}$ & $3.61 \mathrm{a}$ & 7.949 & 0 \\
Q3 & $4.54 \mathrm{a}$ & $4.66 \mathrm{ab}$ & $4.83 \mathrm{~b}$ & 4.133 & 0.017 \\
Q4 & $4.59 \mathrm{ab}$ & $4.51 \mathrm{a}$ & $4.75 \mathrm{~b}$ & 3.799 & 0.023 \\
Q5 & $4.38 \mathrm{a}$ & $4.37 \mathrm{a}$ & $4.47 \mathrm{a}$ & 0.64 & 0.528 \\
Q6 & $4.17 \mathrm{~b}$ & $3.95 \mathrm{ab}$ & $3.83 \mathrm{a}$ & 2.182 & 0.114 \\
Q7 & $4.43 \mathrm{ab}$ & $4.36 \mathrm{a}$ & $4.63 \mathrm{~b}$ & 4.185 & 0.016 \\
Q8 & $4.62 \mathrm{~b}$ & $4.35 \mathrm{a}$ & $4.48 \mathrm{ab}$ & 3.099 & 0.046 \\
Q9 & $4.10 \mathrm{~b}$ & $4.31 \mathrm{ab}$ & $3.70 \mathrm{a}$ & 2.476 & 0.085 \\
Q10 & $4.19 \mathrm{a}$ & $4.31 \mathrm{a}$ & 0.456 & 0.634 \\
Q11 & $4.56 \mathrm{a}$ & $4.55 \mathrm{a}$ & $4.68 \mathrm{a}$ & 1.23 & 0.293 \\
Q12 & $4.27 \mathrm{~b}$ & $3.96 \mathrm{ab}$ & $3.99 \mathrm{a}$ & 1.692 & 0.185 \\
Q13 & $3.78 \mathrm{a}$ & $3.78 \mathrm{a}$ & 1.54 & 0.216 \\
Q14 & $4.00 \mathrm{a}$ & $4.02 \mathrm{a}$ & $4.07 \mathrm{a}$ & 0.137 & 0.872 \\
Q15 & $3.60 \mathrm{a}$ & $3.61 \mathrm{a}$ & $3.60 \mathrm{a}$ & 0.005 & 0.995 \\
Q16 & $4.35 \mathrm{~b}$ & $4.02 \mathrm{a}$ & $4.16 \mathrm{ab}$ & 3.461 & 0.032 \\
Q17 & $4.11 \mathrm{a}$ & $3.97 \mathrm{a}$ & $4.22 \mathrm{a}$ & 2.347 & 0.097 \\
Q18 & $3.41 \mathrm{~b}$ & $3.15 \mathrm{ab}$ & $2.85 \mathrm{a}$ & 3.929 & 0.02 \\
Q19 & $3.86 \mathrm{a}$ & $3.88 \mathrm{a}$ & $3.97 \mathrm{a}$ & 0.283 & 0.754 \\
Q20 & $4.57 \mathrm{a}$ & $4.44 \mathrm{a}$ & $4.60 \mathrm{a}$ & 1.632 & 0.197 \\
Q21 & $4.02 \mathrm{a}$ & $3.74 \mathrm{a}$ & $3.73 \mathrm{a}$ & 1.829 & 0.162 \\
\hline
\end{tabular}

Letters $\mathrm{a}, \mathrm{b}$ denote similarities and differences based on variance analysis. Same letter denotes similarity, different letters denote difference (Post Hoc Test, Duncan, alpha 0.05) 
Table 6. Mean preference and significance levels of questioned qualities based on participant occupation

\begin{tabular}{c|c|c|c|c|c|c}
\hline \multirow{2}{*}{ Qualities } & \multicolumn{7}{|c}{ Occupation } \\
\cline { 2 - 7 } & Student & Civil servant & $\begin{array}{c}\text { Self emp./Private } \\
\text { sector employee }\end{array}$ & Others & F & Sig. \\
\hline Q1 & $3.98 \mathrm{a}$ & $3.93 \mathrm{a}$ & $3.96 \mathrm{a}$ & $4.08 \mathrm{a}$ & 0.352 & 0.788 \\
Q2 & $4.22 \mathrm{~b}$ & $3.78 \mathrm{a}$ & $3.70 \mathrm{a}$ & $3.93 \mathrm{a}$ & 6.387 & 0 \\
Q3 & $4.67 \mathrm{a}$ & $4.67 \mathrm{a}$ & $4.68 \mathrm{a}$ & $4.73 \mathrm{a}$ & 0.178 & 0.911 \\
Q4 & $4.52 \mathrm{a}$ & $4.58 \mathrm{a}$ & $4.62 \mathrm{a}$ & $4.63 \mathrm{a}$ & 0.519 & 0.67 \\
Q5 & $4.46 \mathrm{ab}$ & $4.31 \mathrm{a}$ & $4.54 \mathrm{~b}$ & $4.28 \mathrm{a}$ & 2.262 & 0.081 \\
Q6 & $4.08 \mathrm{a}$ & $3.88 \mathrm{a}$ & $3.82 \mathrm{a}$ & $3.99 \mathrm{a}$ & 1.466 & 0.223 \\
Q7 & $4.41 \mathrm{a}$ & $4.34 \mathrm{a}$ & $4.50 \mathrm{a}$ & $4.51 \mathrm{a}$ & 1.047 & 0.372 \\
Q8 & $4.38 \mathrm{a}$ & $4.48 \mathrm{a}$ & $4.36 \mathrm{a}$ & $4.44 \mathrm{a}$ & 0.501 & 0.682 \\
Q9 & $3.89 \mathrm{ab}$ & $3.67 \mathrm{a}$ & $4.08 \mathrm{~b}$ & $3.74 \mathrm{a}$ & 2.546 & 0.056 \\
Q10 & $4.32 \mathrm{a}$ & $4.30 \mathrm{a}$ & $4.28 \mathrm{a}$ & $4.23 \mathrm{a}$ & 0.224 & 0.88 \\
Q11 & $4.54 \mathrm{a}$ & $4.60 \mathrm{a}$ & $4.57 \mathrm{a}$ & $4.63 \mathrm{a}$ & 0.32 & 0.811 \\
Q12 & $4.14 \mathrm{a}$ & $4.11 \mathrm{a}$ & $3.95 \mathrm{a}$ & $4.05 \mathrm{a}$ & 0.762 & 0.516 \\
Q13 & $4.07 \mathrm{~b}$ & $3.81 \mathrm{ab}$ & $3.75 \mathrm{a}$ & $3.84 \mathrm{ab}$ & 2.241 & 0.083 \\
Q14 & $4.05 \mathrm{a}$ & $4.03 \mathrm{a}$ & $4.04 \mathrm{a}$ & $3.97 \mathrm{a}$ & 0.154 & 0.927 \\
Q15 & $3.53 \mathrm{a}$ & $3.58 \mathrm{a}$ & $3.72 \mathrm{a}$ & $3.67 \mathrm{a}$ & 0.666 & 0.574 \\
Q16 & $4.01 \mathrm{a}$ & $4.18 \mathrm{a}$ & $4.14 \mathrm{a}$ & $4.10 \mathrm{a}$ & 0.91 & 0.436 \\
Q17 & $4.04 \mathrm{a}$ & $4.09 \mathrm{a}$ & $4.00 \mathrm{a}$ & $4.03 \mathrm{a}$ & 0.14 & 0.936 \\
Q18 & $3.35 \mathrm{a}$ & $2.98 \mathrm{a}$ & $3.05 \mathrm{a}$ & $3.00 \mathrm{a}$ & 2.399 & 0.067 \\
Q19 & $4.06 \mathrm{a}$ & $3.73 \mathrm{a}$ & $3.86 \mathrm{a}$ & $3.90 \mathrm{a}$ & 2.085 & 0.101 \\
Q20 & $4.45 \mathrm{a}$ & $4.51 \mathrm{a}$ & $4.50 \mathrm{a}$ & $4.53 \mathrm{a}$ & 0.229 & 0.876 \\
Q21 & $3.78 \mathrm{a}$ & $3.71 \mathrm{a}$ & $3.83 \mathrm{a}$ & $3.83 \mathrm{a}$ & 0.289 & 0.834 \\
\hline
\end{tabular}

Letters $\mathrm{a}, \mathrm{b}$ denote similarities and differences based on variance analysis. Same letter denotes similarity, different letters denote difference (Post Hoc Test, Duncan, alpha 0.05)

Table 7. Mean preference and significance levels of questioned qualities based on participant income

\begin{tabular}{|c|c|c|c|c|c|c|c|c|}
\hline \multirow{2}{*}{ Qualities } & \multicolumn{8}{|c|}{ Income (TL) } \\
\hline & No income & $500-1500$ & $1600-2500$ & $2600-3500$ & $3600-4500$ & $>4600$ & $\mathbf{F}$ & Sig. \\
\hline Q1 & $4.09 \mathrm{a}$ & $3.90 \mathrm{a}$ & $4.06 \mathrm{a}$ & $3.96 \mathrm{a}$ & $3.95 \mathrm{a}$ & $3.81 \mathrm{a}$ & 0.776 & 0.568 \\
\hline Q2 & $4.19 \mathrm{c}$ & $4.10 \mathrm{c}$ & $4.12 \mathrm{c}$ & $3.94 \mathrm{bc}$ & $3.68 \mathrm{ab}$ & $3.46 \mathrm{a}$ & 6.674 & 0 \\
\hline Q3 & $4.66 \mathrm{ab}$ & $4.63 \mathrm{ab}$ & $4.82 \mathrm{~b}$ & $4.53 \mathrm{a}$ & $4.77 \mathrm{ab}$ & $4.69 \mathrm{ab}$ & 1.432 & 0.211 \\
\hline Q4 & $4.55 \mathrm{a}$ & $4.52 \mathrm{a}$ & $4.67 \mathrm{a}$ & $4.70 \mathrm{a}$ & $4.49 \mathrm{a}$ & $4.60 \mathrm{a}$ & 0.781 & 0.564 \\
\hline Q5 & $4.42 \mathrm{~b}$ & $4.50 \mathrm{~b}$ & $4.49 \mathrm{~b}$ & $4.55 \mathrm{~b}$ & $4.08 \mathrm{a}$ & $4.41 \mathrm{~b}$ & 3.126 & 0.009 \\
\hline Q6 & $4.05 \mathrm{bc}$ & $4.29 \mathrm{c}$ & $4.02 \mathrm{bc}$ & $3.98 \mathrm{bc}$ & $3.81 \mathrm{ab}$ & $3.60 \mathrm{a}$ & 3.324 & 0.006 \\
\hline Q7 & $4.38 \mathrm{ab}$ & $4.56 \mathrm{~b}$ & $4.53 \mathrm{~b}$ & $4.60 \mathrm{~b}$ & $4.19 \mathrm{a}$ & $4.47 \mathrm{ab}$ & 2.423 & 0.035 \\
\hline Q8 & $4.35 \mathrm{a}$ & $4.54 \mathrm{a}$ & $4.51 \mathrm{a}$ & $4.57 \mathrm{a}$ & $4.35 \mathrm{a}$ & $4.34 \mathrm{a}$ & 1.027 & 0.401 \\
\hline Q9 & $3.87 \mathrm{ab}$ & $4.00 \mathrm{~b}$ & $3.73 \mathrm{ab}$ & $4.06 \mathrm{~b}$ & $3.55 \mathrm{a}$ & $3.79 \mathrm{ab}$ & 1.712 & 0.131 \\
\hline Q10 & $4.30 \mathrm{a}$ & $4.31 \mathrm{a}$ & $4.29 \mathrm{a}$ & $4.43 \mathrm{a}$ & $4.27 \mathrm{a}$ & $4.15 \mathrm{a}$ & 0.656 & 0.657 \\
\hline Q11 & $4.59 \mathrm{a}$ & $4.42 \mathrm{a}$ & $4.63 \mathrm{a}$ & $4.62 \mathrm{a}$ & $4.59 \mathrm{a}$ & $4.57 \mathrm{a}$ & 0.607 & 0.695 \\
\hline Q12 & $4.08 \mathrm{abc}$ & $4.29 \mathrm{c}$ & $4.22 \mathrm{abc}$ & $4.26 \mathrm{bc}$ & $3.86 \mathrm{a}$ & $3.91 \mathrm{ab}$ & 2.282 & 0.046 \\
\hline Q13 & $3.99 \mathrm{a}$ & $4.00 \mathrm{a}$ & $3.92 \mathrm{a}$ & $3.91 \mathrm{a}$ & $3.70 \mathrm{a}$ & $3.78 \mathrm{a}$ & 1.005 & 0.414 \\
\hline Q14 & $4.03 \mathrm{a}$ & $4.06 \mathrm{a}$ & $3.98 \mathrm{a}$ & $4.13 \mathrm{a}$ & $3.99 \mathrm{a}$ & $4.00 \mathrm{a}$ & 0.192 & 0.966 \\
\hline Q15 & $3.49 \mathrm{a}$ & $3.60 \mathrm{a}$ & $3.82 \mathrm{a}$ & $3.64 \mathrm{a}$ & $3.68 \mathrm{a}$ & $3.59 \mathrm{a}$ & 0.81 & 0.543 \\
\hline Q16 & $3.94 \mathrm{a}$ & $3.94 \mathrm{a}$ & $4.45 \mathrm{c}$ & $4.36 \mathrm{bc}$ & $4.05 \mathrm{ab}$ & $4.16 \mathrm{abc}$ & 3.662 & 0.003 \\
\hline Q17 & $3.99 \mathrm{a}$ & $4.06 \mathrm{a}$ & $4.00 \mathrm{a}$ & $4.19 \mathrm{a}$ & $3.95 \mathrm{a}$ & $4.21 \mathrm{a}$ & 0.797 & 0.552 \\
\hline Q18 & $3.11 \mathrm{ab}$ & $3.79 \mathrm{c}$ & $3.33 \mathrm{~b}$ & $3.06 \mathrm{ab}$ & $2.97 \mathrm{ab}$ & $2.69 \mathrm{a}$ & 4.962 & 0 \\
\hline Q19 & $3.99 \mathrm{a}$ & $4.06 \mathrm{a}$ & $3.80 \mathrm{a}$ & $3.89 \mathrm{a}$ & $3.78 \mathrm{a}$ & $3.76 \mathrm{a}$ & 0.868 & 0.503 \\
\hline Q20 & $4.50 \mathrm{a}$ & $4.52 \mathrm{a}$ & $4.47 \mathrm{a}$ & $4.66 \mathrm{a}$ & $4.45 \mathrm{a}$ & $4.38 \mathrm{a}$ & 0.71 & 0.616 \\
\hline Q21 & $3.82 \mathrm{ab}$ & $3.85 \mathrm{ab}$ & $3.84 \mathrm{ab}$ & $4.09 \mathrm{~b}$ & $3.54 \mathrm{a}$ & $3.59 \mathrm{a}$ & 2.223 & 0.051 \\
\hline
\end{tabular}

Letters $\mathrm{a}, \mathrm{b}, \mathrm{c}$ denote similarities and differences based on variance analysis. Same letter denotes similarity, different letters denote difference (Post Hoc Test, Duncan, alpha 0.05) 


\section{Discussion and conclusions}

Schirpke et al. (2018) reported that social preferences could be explained by landscaping properties and tourism infrastructure. In fact, the primary aim of the present study was to determine the correlation between individual preferences for rural recreation areas and the landscape qualities. Several studies were conducted on the expectations/preferences of individuals about recreation areas (e.g., Cheung and Jim, 2013; Chiu et al., 2016; Heagney et al., 2018). The present study is not a field study, but it is about the qualities that determined the rural recreational area preferences of the individuals.

The way individuals spend leisure time is, above all, closely associated with certain demographics. Thus, participants' gender, age, occupation, education and income level data were scrutinized in the study.

The rural recreation area satisfaction factors vary based on occupant demographics. In a study conducted by Uzun and Müderrisoğlu (2010), it was partially confirmed that rural recreation area satisfaction factors varied based on user demographics and the user demographics such as age, education level, time spent in the area, visit frequency and group size were effective on rural recreation area satisfaction. In the present study, analysis of participant preference scores for 21 qualities demonstrated that the highest difference (significant difference) was based on differences in gender, education and income levels (for 10 qualities). This was followed by age (7 qualities) and occupation (4 qualities). Participant demographics also affected the preference rankings.

In certain studies conducted with couples, it was found that there was no significant difference between the leisure satisfaction levels based on gender (e.g., Siegenthaller and O'Dell, 2000; Di Bona, 2000; Berg et al., 2001). In the present study, it was determined that there was a significant difference between 10 quality scores.

The most preferred experiences include enjoying the natural scenery, peace and quiet. These qualities are preferred preferably near the home of the visitors; however, these are increasingly found only in rural and suburban areas (Bell et al., 2007). In the present study, it was observed that the most important quality for the potential visitors was "being in contact with the nature, facilities to rest, relaxation, and to have a peace of mind" (Q3).

Previous studies in the literature reported that besides the natural characteristics of rural recreation areas (such as unique landscapes and locations), other factors that affect the visitor demand include weather conditions (Humpel et al., 2002; Suminski et al., 2008; Nasir et al., 2012), accessibility (Humpel et al., 2002; Neuvonen et al., 2007; Bestard and Font, 2009; Paracchini et al., 2014; Ala-Hulkko et al., 2016), facilities, price and quality of services (Sava, 2015), accommodation and periodical lodging facilities (Humpel et al., 2002), peer recommendation, advertising-promotions (Sava, 2015), and security and aesthetic features (Humpel et al., 2002). Furthermore, Folmer et al. (2016) investigated the reasons for attractiveness and participant descriptions for the most attractive green spaces. In conclusion, it was determined that qualities such as large green areas, silence, naturalness, water (attractive water surface, river, lake or sea), panoramic and open views, good recreational opportunities, diversity (diverse plant species, land use and seasons), non-urban characteristics, personal bond, historical characteristics, wildlife and flora, harmony (of the landscape), farming (the landscape used by farmers) were effective on occupant preferences. The qualities scrutinized in the present study are consistent with previous study findings. 
Recreational and aesthetic values generally overlap and these are usually discussed together (Van Zanten et al., 2016). For example, a beautiful landscape is an important factor in tourism destination and nature-based recreation preferences (Scolozzi et al., 2014). The high landscape quality and unspoiled nature in rural recreation areas are among the most sought-after qualities by potential visitors, as demonstrated by Q4, Q10 and Q11.

On the other hand, urbanization and high number of visitors negatively affect recreation quality (Scolozzi et al., 2014). The present study findings demonstrated that festivals, festivities, concerts, etc. organized in the recreation area were moderately or relatively effective (Q18, MPS: 3.12) on visitor preferences. However, the calmness of the recreation area, the lack of crowds (Q17, MPS: 4.05) were more effective factors on preference.

Certain studies reported that cultural preferences might affect park occupancy (Tu et al., 2015; Chow et al., 2016). Public spaces and recreation facilities offer a variety of facilities for children, adults and families, however activity preferences in parks depend on the occupant age and gender (Cohen et al., 2007; Kaczynski et al., 2011; Moore et al., 2017). Thus, alternatives should be designed based on different user requirements, or thematic approaches should be preferred in plans.

Accessible infrastructure determines the occupancy of a suitable recreational area and proximity to residential areas is a crucial factor for recreational area occupancy (Weyland and Laterra, 2014; Peña et al., 2015; Ala- Hulkko et al., 2016; Schirpke et al., 2018). Certain studies demonstrated that there was a correlation between the distance to the recreation area and the number of visits to the recreation area; the number of visits decreased with an increase in the distance to the recreation area or the nearest forest (Roovers et al., 2002; Neuvonen et al., 2007). However, it was observed that one of the most significant preference factors was proximity to home parameter (e.g. Sava, 2015). Furthermore, there was a significant correlation between accessibility, facilities, and aesthetic attributes and physical activity (Neuvonen et al., 2007). However, the present study findings demonstrated that "easy access to the recreation area" (Q1, MPS = 3.98) was an effective quality and among the most preferred 8 qualities. This may be due to availability of natural resources in the city of the participants' residence, personal preferences and other factors.

Individuals prefer forests, bodies of water and natural mountain landscapes for wildlife observation, hiking and trekking activities (Bell et al., 2007; Pastorella et al., 2017). Most rural recreation areas include forests. The presence of recreational facilities such as picnic areas, training paths and parking lots could affect the recreational preferences in forests (Tapsuwan et al., 2012; Agimass et al., 2018). Furthermore, the presence of various landscape characteristics in or near the recreation areas (such as different landscapes, water and coastal areas) increases the value of the area for complementary recreational activities. Previous studies demonstrated that the presence of water leads to high landscape preference scores (Kaltenborn and Bjerke, 2002; Dramstad et al., 2006). Kaplan and Kaplan (1989) reported that this preference may be due to the evolutionary adaptation of humankind (De Valck et al., 2017). In fact, it was determined that Q14 was "very effective" (MPS $=4.03$ ) on rural recreation area preference.

In visitor decision-making processes, peer recommendations are more effective when compared to mass media promotion of services (Sava, 2015). It was determined that previous experiences and satisfaction were an effective factor on repeat visits in these 
areas $(\mathrm{Q} 21, \mathrm{MPS}=3.78)$. On the other hand, the fact that the facilities available in the recreation area that allow the visitors to spend time with family and friends (Q8, MPS $=4.42)$ was preferred more when compared the ability to spend personal time $(\mathrm{Q} 9$, MPS $=3.83$ ). This may be due to the personal or cultural traits of the participants.

The present study findings were relatively parallel to the findings of similar studies conducted in various countries. However, the present study findings demonstrated that the 8 qualities were more effective on visitor preferences about a rural recreation area. These qualities were also the most influential qualities for a rural recreation area to attract visitors. On the other hand, it was noted that there were significant differences between the preference scores based on participant demographics.

Unspoiled rural landscape without human intervention and related natural beauties decrease every day and the importance of these areas increase gradually. Thus, sustainability of natural resources is one of the important targets for nature-based tourism destinations. It is important to understand the expectations of the occupants and to take management decisions accordingly (Lime et al., 2004). In order to achieve a broader perspective in the measurement of the demand for recreation areas, it is necessary to investigate the factors that affect the preferences and tendencies of potential visitors with a multidimensional approach (such as social and cultural traits, service providers, local administrations). It was observed that the qualities discussed in the present study had significant effects on rural recreation preferences. Therefore, the present study could contribute to the future planning strategies of the decision-makers and further comprehensive studies.

Acknowledgements. The author would like to thank Artvin Coruh University for their consent and all participants for their contribution to the study. The experimental section of the present study was previously presented in the XI. European Conference on Social and Behavioral Sciences (2016, Rome, Italy).

\section{REFERENCES}

[1] Agimass, F., Lundhede, T., Panduro, T. E., Jacobsen, J. B. (2018): The choice of forest site for recreation: A revealed preference analysis using spatial data. - Ecosystem Services 31(C): 445-454.

[2] Ala-Hulkko, T., Kotavaara, O., Alahuhta, J., Helle, P., Hjort, J. (2016): Introducing accessibility analysis in mapping cultural ecosystem services. - Ecological Indicators 66: 416-427.

[3] Andkjær, S., Arvidsen, J. (2015): Places for active outdoor recreation - a scoping review. - Journal of Outdoor Recreation and Tourism 12: 25-46.

[4] Beeco, J. A., Hallo, J. C., Brownlee, M. T. (2014): GPS visitor tracking and recreation suitability mapping: tools for understanding and managing visitor use. - Landscape and Urban Planning 127: 136-145.

[5] Bell, S., Tyrväinen, L., Sievänen, T., Pröbstl, U., Simpson, M. (2007): Outdoor recreation and nature tourism: a European perspective. - Living Reviews in Landscape Research 1(2): 1-46.

[6] Berg, E., Trost, M., Schneider, I. E., Allison, M. T. (2001): Dyadic exploration of the relationship of leisure satisfaction, leisure time, and gender to relationship satisfaction. Leisure Sciences 23: 35-46.

[7] Bestard, A. B., Font, A. R. (2009): Environmental diversity in recreational choice modeling. - Ecological Economics 68: 2743-2750. 
[8] Cheung, L. T. O., Jim, C. Y. (2013): Ecotourism service preference andmanagement in Hong Kong. - International Journal of Sustainable Development and World Ecology 20(2): 182-194.

[9] Chiu, H. Y., Chan, C. S., Marafa, L. M. (2016): Local perception and preferences in nature tourism in Hong Kong. - Tourism Management Perspectives 20: 87-97.

[10] Chow, B. C., McKenzie, T. L., Sit, C. H. P. (2016): Public parks in Hong Kong: characteristics of physical activity areas and their users. - International Journal of Environmental Research and Public Health 13(7): 639.

[11] Cohen, D. A., McKenzie, T. L., Sehgal, A., Williamson, S., Golinelli, D., Lurie, N. (2007): Contribution of public parks to physical activity. - American Journal of Public Health 97(3): 509-514.

[12] Cordell, H. K., Betz, C. J., Green, G. T. (2002): Recreation and the environment as cultural dimensions in contemporary American society. - Leisure Science 24: 13-41.

[13] Cox, D. T. C., Shanahan, D. F., Hudson, H. L., Plummer, K. E, Siriwardena, G. M., Fuller, R. A., Anderson, K., Hancock, S., Gaston, K. J. (2017): Doses of neighborhood nature: The benefits for mental health of living with nature. - BioScience 67(2): 147-155.

[14] De Valck, J., Broekx, S., Liekens, I., De Nocker, L., Van Orshoven, J., Vranken, L. (2016): Contrasting collective preferences for outdoor recreation and substitutability of nature areas using hot spot mapping. - Landscape and Urban Planning 151: 64-78.

[15] De Valck, J., Landuyt, D., Broekx, S., Liekens, I., De Nocker, L., Vranken, L. (2017): Outdoor recreation in various landscapes: Which site characteristics really matter? - Land Use Policy 65: 186-197.

[16] Di Bona, L. (2000): What are the benefits of leisure? An exploration using the leisure satisfaction scale. - British Journal of Occupational Therapy 63(2): 50-58.

[17] Dramstad, W. E., Tveit, M. S., Fjellstad, W. J., Fry, G. L. A. (2006): Relationships between visual landscape preferences and map-based indicators of landscape structure. Landscape and Urban Planning 78(4): 465-474.

[18] Eminağaoğlu, Ö., Beğen, H. A. (2015): Artvin'de Önemli Hassas Alanlar. - In: Eminağaoğlu, Ö. (ed.) Artvin'in Bitkileri, Promat Basın Yayın San. ve Tic. A.Ş., İstanbul.

[19] Eriksson, L., Nordlund, A. (2013): How is setting preference related to intention to engage in forest recreation activities? - Urban Forestry \& Urban Greening 12: 481-489.

[20] Fleming, C. M., Cook, A. (2008): The recreational value of Lake McKenzie, Fraser Island: An application of the travel cost method. - Tourism Management 29: 1197-1205.

[21] Folmer, A., Haartsen, T., Buijs, A., Huigen, P. P. P. (2016): Wildlife, flora, and the perceived attractiveness of green places: a comparison between local and national green places. - Journal of Outdoor Recreation and Tourism 16: 16-23.

[22] Grêt-Regamey, A., Weibel, B., Kienast, F., Rabe, S., Zulian, G. (2015): A tiered approach for mapping ecosystem services. - Ecosystems Services 13: 16-27.

[23] Guo, Z., Zhang, L., Li, Y. (2010): Increased dependence of humans on ecosystem services and biodiversity. - PloSOne 5(10): e13113.

[24] Hansen-Moller, J., Oustrup, L. (2004): Emotional, physical/ functional and symbolic aspects of an urban forest in Denmark to nearby residents. - Scandinavian Journal of Forest Research 19: 56-64.

[25] Hartig, T., Mitchell, R., de Vries, S., Frumkin, H. (2014): Nature and health. - Annual Review of Public Health 35: 207-228.

[26] Heagney, E. C., Rose, J. M., Ardeshiri, A., Kovac, M. (2018): Optimising recreation services from protected areas: Understanding the role of natural values, built infrastructure and contextual factors. - Ecosystem Services 31: 358-370.

[27] Humpel, N., Owen, N., Leslie, E. (2002): Environmental factors associated with adults' participation in physical activity: a review. - American Journal of Preventive Medicine 22: 188-199. 
[28] Jim, C. Y., Zhang, H. (2013): Species diversity and spatial differentiation of old-valuable trees in urban Hong Kong. - Urban Forestry \& Urban Greening 12: 171-182.

[29] Kaczynski, A. T., Wilhelm Stanis, S. A., Hastmann, T. J., Besenyi, G. M. (2011): Variations in observed park physical activity intensity level by gender, race, and age: individual and joint effects. - Journal of Physical Activity \& Health 8(2): 151-160.

[30] Kaltenborn, B. P., Bjerke, T. (2002): Associations between environmental value orientations and landscape preferences. - Landscape and Urban Planing 59(1): 1-11.

[31] Kaplan, R., Kaplan, S. (1989): The Experience of Nature: A Psychological Perspective. Cambridge University Press, Cambridge, UK.

[32] Korpela, K., Borodulin, K., Neuvonen, M., Paronen, O., Tyrväinen, L. (2014): Analyzing the mediators between nature-based outdoor recreation and emotional well-being. Journal of Environmental Psychology 37: 1-7.

[33] Kraus, R. (1971): Recreation and Leisure in Modern Society. - TACC, USA.

[34] Lee, C. F., Huang, H. I., Yeh, H. R. (2010): Developing an evaluation model for destination attractiveness: Sustainable forest recreation tourism in Taiwan. - Journal of Sustainable Tourism 18(6): 811-828.

[35] Lime, D. W., Anderson, D. H., Thompson, J. L. (2004): Identifying and Monitoring Indicators of Visitor Experience and Resource Quality: A Handbook for Recreation Resource Managers. - University of Minnesota, Department of Forest Resources, St Paul, Minnesota.

[36] McCormack, G. R., Rock, M., Toohey, A. M., Hignell, D. (2010): Characteristics of urban parks associated with park use and physical activity: are view of qualitative research. - Health \& Place 16(4): 712-726.

[37] Moore, J. B., Cook, A., Schuller, K., Lub, Y., Yuan, Z., Maddock, J. E. (2017): Physical activity and park use of youth in Nanchang, China. - Preventive Medicine Reports 8: 256-260.

[38] Nasir, R. A., Anuar, A. N. A., Md Darus, F., Jaini, N., Salleh, S. A., (2012): The climatology effects on outdoor recreation perception and activity in Shah Alam. Procedia Social and Behavioral Sciences 49: 193-201.

[39] Neuvonen, M., Sievänen, T., Tönnes, S., Koskela, T. (2007): Access to green areas and the frequency of visits: a case study in Helsinki. - Urban Forestry and Urban Greening 6: 235-247.

[40] Nielsen, A. B., Olsen, S. B., Lundhede, T. (2007): An economic valuation of the recreational benefits associated with nature-based forest management practices. Landscape and Urban Planning 80: 63-71.

[41] Paracchini, M. L., Zulian, G., Kopperoinen, L., Maes, J., Schägner, J. P., Termansen, M., Zandersen, M., Perez-Soba, M., Scholefield, P. A., Bidoglio, G. (2014): Mapping cultural ecosystem services: A framework to assess the potential for outdoor recreation across the EU. - Ecological Indicators 45: 371-385.

[42] Pastorella, F., Giacovelli, G., De Meo, I., Paletto, A. (2017): People's preferences for Alpine forest landscapes: results of an internet-based survey. - Jornal of Forest Research 22(1): 36-43.

[43] Peña, L., Casado-Arzuaga, I., Onaindia, M. (2015): Mapping recreation supply and demand using an ecological and a social evaluation approach. - Ecosystem Services 13: 108-118.

[44] Plieninger, T., Dijks, S., Oteros-Rozas, E., Bieling, C. (2013): Assessing, mapping, and quantifying cultural ecosystem services at community level. - Land Use Policy 33: 118129.

[45] Roovers, P., Hermy, M., Gulick, H. (2002): Visitor profile, perceptions and expectations in forests from a gradient of increasing urbanization in Belgium. - Landscape and Urban Planning 59: 129-145. 
[46] Rosenberger, R. S., Needham, M. D., Morzillo, A. T., Moehrke, C. (2012): Attitudes, willingness to pay, and stated values for recreation use fees at an urban proximate forest. - Journal of Forest Economics 18: 271-281.

[47] Şahbaz, R. P., Altınay, M. (2015): Türkiye'deki milli parkların rekreasyon faaliyetleri açısından değerlendirilmesi. - Journal of Tourism and Gastronomy Studies 3(3): 125-135.

[48] Sanagustin Fons, M. V., Moseñe Fierro, J. A., Gómez y Patiño, M. (2011): Rural tourism: A sustainable alternative. - Applied Energy 88: 551-557.

[49] Sava, A. M. (2015): Factors affecting the choice of recreation providers: a conceptual model. - Procedia Economics and Finance 23: 622-627.

[50] Schirpke, U., Meisch, C., Marsoner, T., Tappeiner, U. (2018): Revealing spatial and temporal patterns of outdoor recreation in the European Alps and their surroundings. Ecosystem Services 31: 336-350.

[51] Scolozzi, R., Schirpke, U., Detassis, C., Abdullah, S., Gretter, A. (2014): Mapping alpine landscape values and related threats as perceived by tourists. - Landscape Research 40(4): 1-15.

[52] Shrestha, R. K., Stein, T. V., Clark, J. (2007): Valuing nature-based recreation in public natural areas of the Apalachicola River region, Florida. - Journal of Environmental Management 85: 977-985.

[53] Siegenthaller, K. L., O’Dell, I. (2000): Leisure attitude, leisure satisfaction and perceived freedom in leisure within family dyads. - Leisure Sciences 22: 281-295.

[54] Suminski, R., Poston, W., Market, P., Hyder, M., Sara, P. (2008): Meteorological conditions are associated with physical activities performed in open-air settings. International Journal of Biometeorology 52(3): 189-197.

[55] Tapsuwan, S., Macdonald, D. H., King, D., Poudyal, N. (2012): A combined site proximity and recreation index approach to value natural amenities: an example from a natural resource management region of Murray-Darling Basin. - Journal of Environmental Management 94(1): 69-77.

[56] Tekin, M., Devecioglu, S., Yarar, D. N. (2012): Examination of recreation levels of university students with regard to various variables. - Procedia, Social and Behavioral Sciences 46: 320-325.

[57] Torkildsen, G. (2005): Leisure and Recreation Management. - (5 $5^{\text {th }}$ ed.), NY: Routledge, New York.

[58] Tribe, J. (2011): The Economics of Recreation, Leisure and Tourism. $-\left(4^{\text {th }}\right.$ ed.), Oxford, Elsevier.

[59] Triguero-Mas, M., Dadvand, P., Cirach, M., Martínez, D., Medina, A., Mompart, A., Basagaña, X., Gražulevičienè, R., Nieuwenhuijsen, M. J. (2015): Natural outdoor environments and mental and physical health: relationships and mechanisms. Environment International 77: 35-41.

[60] Tu, H., Liao, X., Schuller, K., Cook, A., Fan, S., Lan, G., Lu, Y., Yuan, Z., Moore, J. B., Maddock, J. E. (2015): Insights from an observational assessment of park-based physical activity in Nanchang, China. - Preventive Medicine Reports 2: 930-934.

[61] Uzun, S., Müderrisoğlu, H. (2010): Kırsal rekreasyon alanlarında kullanıcı memnuniyeti: Bolu Gölcük ormaniçi dinlenme yeri örneği. - Süleyman Demirel Üniversitesi Orman Fakültesi Dergisi 1: 67-82.

[62] van Zanten, B. T., Zasada, I., Koetse, M. J., Ungaro, F., Häfner, K., Verburg, P. H. (2016): A comparative approach to assess the contribution of landscape features to aesthetic and recreational values in agricultural landscapes. - Ecosystem Services 17: 8798.

[63] Weyland, F., Laterra, P. (2014): Recreation potential assessment at large spatial scales: a method based in the ecosystem services approach and landscape metrics. - Ecological Indicators 39: 34-43.

[64] Yang, Y. (2017): The practice and exploration of Shanghai recreational trail system planning. - Procedia Engineering 198: 127-138. 
[65] Zoderer, B. M., Tasser, E., Erb, K., Stanghellini, P. S. L., Tappeiner, U. (2016): Identifying and mapping the tourists' perception of cultural ecosystem services: A case study from an Alpine region. - Land Use Policy 56: 251-261. 